\title{
A Case-Control Study on Male Hepatocellular Carcinoma Based on Hospital and Community Controls
}

\author{
Akira Shibata ', Katsuhiro Fukuda ${ }^{1}$, Atsushi Nishiyori ${ }^{1}$, Itsuro Ogimoto ${ }^{1}$, Ritsu Sakata ${ }^{1}$ \\ and Kyuichi Tanikawa ${ }^{2}$
}

\begin{abstract}
A case control study on male primary hepatocellular carcinoma(HCC) and hepatitis $B$ or $C$ virus and some potential risk factors, e.g. blood transfusion, aldehyde dehydrogenase 2(ALDH2) genotype and drinking habits, was performed using two controls, i.e. a hospital control( $\mathrm{HC})$ and a community control(CC) in Fukuoka and Saga Prefectures. Cases were obtained from the Second Department of Internal Medicine, Kurume University Hospital. The HCs were obtained from inpatients of two general hospitals in Kurume and the CCs were randomly sampled from the Kurume citizens being matched with age and sex to each case. Based on the HCs, odds ratios(ORs) of developing male $\mathrm{HCC}$ were statistically significant due to $\mathrm{HBsAg}$ or anti-HCV antibody positive status. Some discrepancies were observed between the two controls, i.e. higher proportions of past histories of diabetes or hypertension, of ALDH2 typical homozygote(ALDH2 $\left.{ }^{1 / A L D H} 2^{1}\right)$, and of heavy drinkers among the HCs, suggesting slight deviation of the $\mathrm{HCs}$ from the $\mathrm{CCs}$ in alcohol related aspects. Although ORs regarding accumulated amount of alcohol intake by age 40 based on the HCs were insignificant, two of the three corresponding ORs based on the CCs were statistically significant. Judging from alcohol related aspects between the two controls, the ORs for alcohol based on the HCs seems to be underestimated. JEpidemiol, $1998 ; 8: 1-5$.
\end{abstract}

hospital control, community control, hepatitis virus, aldehyde dehydrogenase 2 , drinking habits

Hepatitis $\mathrm{B}$ or $\mathrm{C}$ virus and heavy alcohol drinking have been identified as risk factors for primary hepatocellular carcinoma (HCC) and alcohol has been speculated to be a promoter of HCC but epidemiological result so far is inconclusive. Although a hospital control(HC) in a case control study relating alcohol drinking habits may sometimes be representative of the general population ${ }^{1)}$ and proportional comparability of drinking habits was assumed in the previous study ${ }^{2}$, it might be worthwhile to examine representativeness of a $\mathrm{HC}$ for the general population regarding alcohol related characteristics and relation between alcohol drinking and HCC. Further, sensitivity to alcohol may also be an important factor for developing $\mathrm{HCC}$ and ALDH2 genotype seems to be worthwhile to evaluate the association between the sensitivity and $\mathrm{HCC}$.

The present study compared a HC and a community control(CC) in terms of exposure profile and corresponding odds ratios (ORs) for $\mathrm{HCC}$ by item

\section{MATERIALS AND METHODS}

\section{Case control study}

The study methods are identical to those used in the previous study ${ }^{2}$ except 1) inclusion of one $\mathrm{CC}$ for each case, 2) collection of corresponding information from $\mathrm{CC}$ by a self-administered mailed questionnaire with verification by subsequent telephone interview, 3)exclusion of items on dietary habits and spouse's history of hepatic diseases, and 4)collection of nail clippings for aldehyde dehydrogenase 2 (ALDH2) genotype observation.

Briefly, incident male $\mathrm{HCC}$ cases fulfilling the same criteria of the previous study ${ }^{2)}$ were identified from cases living in either Fukuoka or Saga Prefecture through the records of the

Received January 9, 1997 ; accepted May 2, 1997.

${ }^{1}$ Department of Public Health and 'Second Department of Internal Medicine, Kurume University School of Medicine, 67, Asahimachi, Kurume 830, Japan.

Address for correspondence : Akira Shibata, Department of Public Health, Kurume University School of Medicine, 67 Asahi-machi Kurume, 830 Japan. 
Second Department of Internal Medicine, Kurume University Hospital between June, 1992 and June, 1995. Their age-, sex-, residence-, and time of hospitalization (within 3 months after a case interview)-matched HCs without chronic hepatitis or hepatic cirrhosis were obtained from inpatients of the two general hospitals in Kurume. Their CCs who were 1)Japanese citizens of Kurume, 2)age- and sex-matched to each case within \pm 3 year, and 3)being alive, were randomly sampled from the roster of approximate 230,000 residents at July, 1995. The questionnaire was sent in July, 1995 and all responses were received by early part of October, 1995.

Information on past history including blood transfusion, lifetime smoking habits, lifetime drinking habits and other items of cases and $\mathrm{HCs}$ were obtained through interviews in person by a well-trained interviewer. The lifetime amount of cigarette smoking was quantified by the $\times$ Brinkman-Coates index $t$ which is the product of daily number of cigarettes smoked and years of smoking. The daily amount of alcohol intake was graded by a Xdrink $₹$ defined as the amount of alcoholic beverage containing $23 \mathrm{ml}$ of ethanol. This volume of ethanol is determined because it is the amount of ethanol in one bottle of x chyosif which is one of the Japanese most prevalent unit of quantifying XSeishuE. The accumulated amount of alcohol intake was quantified by $x$ drink-years $€$ which was obtained by multiplying the number of $\ell_{\text {drinks }} £$ by the number of years of consumption as in the previous study ${ }^{2)}$. Hospital records of cases and HCs were reviewed to obtain medical information, e.g. hepatitis B surface antigen ( $\mathrm{HBsAg}$ ) and anti-hepatitis $\mathrm{C}$ virus(anti-HCV) antibody. Information on these factors for CCs was not available.

Nail clippings of cases and $\mathrm{HCs}$ were sampled at the time of interview, and those of CCs were collected at home and mailed to the laboratory in an envelope. ALDH2 genotyping using nail clippings was performed by the method developed by Nishiyori et al. ${ }^{3)}$

Following analyses were conducted only for male. Casecontrol comparison by item was performed by a univariate analysis based on a conditional logistic regression model. The association between selected potential risk factors was evaluated by a chi-square test on $\mathrm{M} \times \mathrm{N}$ table.

Examination of Consistency of Information obtained by Structured Interview and that by Self-administered Mailed Questionnaire

In order to examine consistency of information obtained by structured interview and that by self-administered questionnaire, a questionnaire consisting of the two items, i.e. parental history of hepatic diseases and lifetime drinking habits, identically used by the present structured interview, was constructed. The questionnaire was sent to 22 cases and 30 controls after they received the structured interview between February and May 1996 but they were analyzed separately from the present case-control analysis.

Intraclass correlation coefficient (ICC) was used to examine the extent of the information consistency.

\section{RESULTS}

Between June, 1992 and June, 1995, 989 HCC patients were hospitalized in Kurume University Hospital. Age limit of 4069 was applied to the former period of June, 1992 through December, 1994 and that of 40-74 was applied to the latter period thereafter. Eight hundred and six of them were excluded. Those included 222 having been already interviewed, 180 out of the age-limit, 122 without HCC, 109 living out of the residence-limit, 106 having been diagnosed over 1 year prior to admission, or remainders who were not interviewed from miscellaneous reasons including physician's refusal. None of cases and potential HCs refused to be interviewed but of 203 citizens sampled from the roster $7 \%$ had left the city and $14 \%$ refused cooperation. Finally, 115 male cases, their HCs and CCs were

Table 1. Age Distribution of Study Subjects at July 1, 1995.

\begin{tabular}{lccc}
\hline Age & HCC cases & Hospital controls Community controls \\
\hline $40-49$ & 10 & 10 & 9 \\
$50-59$ & 25 & 29 & 30 \\
$60-69$ & 74 & 70 & 70 \\
$70-74$ & 6 & 6 & 6 \\
\hline Total & $115\left(55^{*}+60^{* *}\right)$ & $115\left(55^{*}+60^{* *}\right)$ & $115^{* * *}$ \\
\hline$*$ & collected between June, 1992 and December, 1994 \\
$* *$ & collected with nail clippings between January and June, 1995 \\
$* * *$ & collected with nail clippings in July, 1995
\end{tabular}

Table 2. Diagnoses at Hospitalization among Hospital Controls.

\begin{tabular}{lrc}
\hline Diagnosis & $\mathrm{N}$ & $\%$ \\
\hline Diabetes mellitus & 34 & 29.6 \\
Malignant neoplasms & 31 & 27.0 \\
$\quad$ Stomach & 10 & 8.7 \\
$\quad$ Lung & 8 & 7.0 \\
$\quad$ Rectum & 4 & 3.5 \\
$\quad$ Colon & 2 & 1.7 \\
$\quad$ Prostate & 2 & 1.7 \\
$\quad$ Others & 5 & 4.3 \\
Gastric ulcer & 6 & 5.2 \\
Prostatic hypertrophy & 6 & 5.2 \\
Benign neoplasms & 3 & 2.6 \\
Asthma & 3 & 2.6 \\
Gallstone & 3 & 2.6 \\
Others* & 29 & 25.2 \\
\hline Total & 115 & 100.0 \\
\hline
\end{tabular}

*Other 27 diagnoses were combined in this category. 
Table 3. Exposure Distribution of Study Subjects and Odds Ratio by Univcariate Conditional Logistic Regression Analysis.

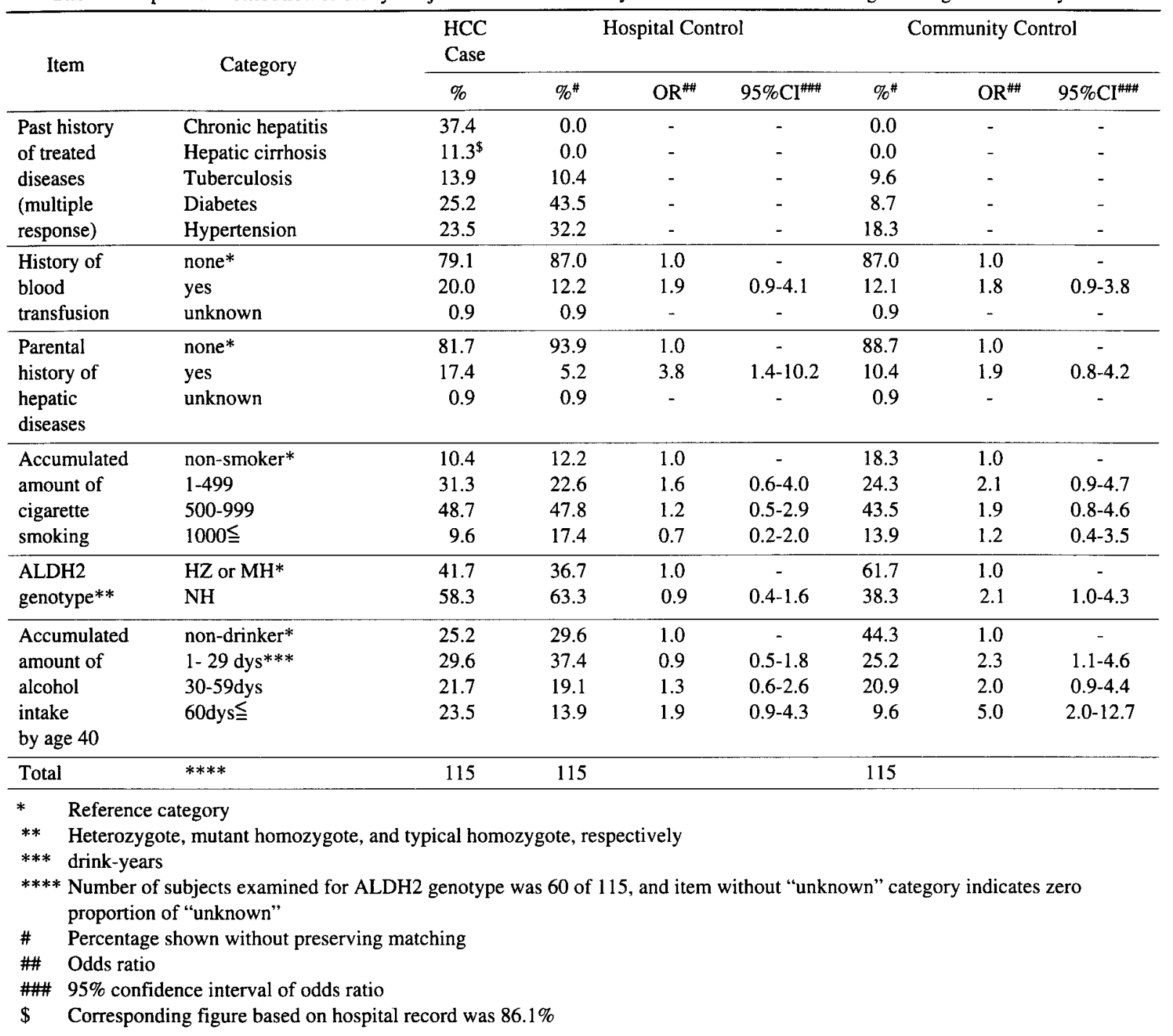

completed.

Age distribution at July 1, 1995 of cases and their HCs combined of the two periods, and of their CCs are shown in Table 1. Approximately $30 \%$ of study subjects had $x_{\text {craftsmen, min- }}$ ing, production process and construction workers and laborers モas the longest occupation and $x_{\text {sales workers }}$ was the next most frequent category. Comparison between the two controls revealed no proportional differences in terms of the longest occupation, most of past histories, e.g. tuberculosis, or gastrointestinal diseases, history of blood transfusion, parental history of hepatic diseases, and accumulated amount of cigarette smoking. The breakdown of diagnoses among HCs at hospitalization is shown in Table 2. The HCs, however, showed larger proportions of past histories of diabetes or hypertension, of ALDH2 typical homozygote, and of heavy drinkers as shown in Table 3.

None of ALDH2 genotype proportion of cases and two controls differ from the Hardy-Weinberg principle.

ORs regarding history of blood transfusion and accumulated amount of cigarette smoking were not statistically significant based on either control. Although none of ORs due to ALDH2 typical homozygote and accumulated amount of alcohol intake 
Table 4. HBsAg and anti-HCV Antibody Status of Study Subjects and Odds Ratio by Univariate Conditional Logistic Regression Analysis.

\begin{tabular}{|c|c|c|c|c|c|}
\hline \multirow[t]{2}{*}{ Item } & \multirow[t]{2}{*}{ Category } & \multirow{2}{*}{$\begin{array}{c}\mathrm{HCC} \\
\text { Case }\end{array}$} & \multicolumn{3}{|c|}{$\begin{array}{c}\text { Hospital } \\
\text { Control }\end{array}$} \\
\hline & & & $\%^{\#}$ & $\mathrm{OR}^{\# \#}$ & $95 \% \mathrm{CI}^{\#}$ \\
\hline \multirow[t]{3}{*}{ HBsAg } & negative* & 87.0 & 99.1 & 1.0 & - \\
\hline & positive & 13.0 & 0.9 & 15.0 & $2.0-113.6$ \\
\hline & unknown & 0.0 & 0.0 & - & - \\
\hline \multirow[t]{3}{*}{ anti-HCV } & negative* & 16.5 & 92.2 & 1.0 & - \\
\hline & positive & 83.5 & 7.0 & 89.0 & $12.4-638.8$ \\
\hline & unknown & 0.0 & 0.9 & - & - \\
\hline \multicolumn{2}{|l|}{ Total } & 115 & 115 & & \\
\hline \multicolumn{6}{|c|}{ * Reference category } \\
\hline \multicolumn{6}{|c|}{ \# Percentage shown without preserving matching } \\
\hline \multicolumn{6}{|c|}{ \#\# Odds ratio } \\
\hline \multicolumn{6}{|c|}{ \#\# 95\% confidence interval of odds ratio } \\
\hline
\end{tabular}

by age 40 based on the HCs were statistically significant, ORs due to ALDH2 typical homozygote and some of accumulated amount of alcohol intake by age 40 based on the CCs were statistically significant as shown in Table 3.

The proportions of positive status of HBsAg and anti-HCV antibody were $13 \%$ and $83.5 \%$ for cases and the corresponding figures were $0.9 \%$ and $7 \%$ for $\mathrm{HCs}$, respectively. ORs of developing $\mathrm{HCC}$ estimated by a univariate conditional logistic regression analysis were 15 and 89 for $\mathrm{HBsAg}$ and anti-HCV antibody positive status, respectively, as shown in Table 4.

Positive associations were observed between factors, i.e. anti-HCV antibody status vs. history of blood transfusion $\left(\chi^{2}=\right.$ 13.29, $\mathrm{df}=2$ ), anti-HCV antibody status vs. parental history of hepatic diseases $\left(\chi^{2}=6.64, \mathrm{df}=1\right)$, and ALDH2 genotype vs. accumulated amount of alcohol intake by age $40\left(\chi^{3}=17.34\right.$, $\mathrm{df}=2$ ).

ICCs for parental history of hepatic diseases and the accumulated amount of alcohol intake by age 40 were 0.922 $(p<0.05)$ and $0.821(p<0.05)$, respectively.

\section{DISCUSSION}

The Japan Primary Liver Cancer Research Group reported profiles of approximately 10,000 Japanese male HCC collected between 1992 and 19934). The most prevalent age group was early sixties. Proportions of positive $\mathrm{HBsAg}$ and anti-HCV antibody and history of blood transfusion were $17.7 \%, 75.4 \%$, and $21.1 \%$, respectively. Cases in the present study show less prevalent positive $\mathrm{HBsAg}$ and more prevalent positive antiHCV antibody.

The CCs for the present study may be regarded as representative of the general population if based on the facts of being sampled at random from the general citizens and similar pro- portion of history of blood transfusion, $12.2 \%$ for CCs vs. $8 \%$ which was observed by the mass-screening for hepatic diseases in Saga Prefecture in $1987^{5}$. The $14 \%$ of refusal among the citizens may reflect less motivation of general population control $^{6)}$. Exposure fractions of HBsAg, $0.9 \%$, and anti-HCV antibody, $7.0 \%$, among the HCs were nearly comparable to $1.7 \%$ and $4-13 \%$, respectively, which were observed by the massscreening ${ }^{5)}$. Assuming high consistency of information obtained by the structured interview with that by the selfadministered questionnaire, which was suggested by the significant positive ICCs for parental history of hepatic diseases and for the accumulated amount of alcohol intake, the HCs may deviate from the general Kurume citizens in alcohol-related aspect, because of a larger proportion of past history of hypertension or diabetes, ALDH2 typical homozygote, and heavy drinker among the HCs.

The findings that 1)quantity-frequency method was regarded as a method of choice for observing drinking habit ${ }^{7}, 2$ ) recalled alcohol intake might be a good predictor of past intake $^{8)}, 3$ ) accumulated amount of alcohol intake by age 40 showed stronger correlation with $\mathrm{HCC}$ in the previous study ${ }^{2}$, 4) applying twice interviews similar to the present method showed satisfactory reproducibility of responses on accumulated amount of drinking by age 40 , i.e. $\mathrm{ICC}=0.788(\mathrm{p}<0.05)^{9}$ ), may support usage of accumulated amount of drinking by age 40 as an index of past drinking habits.

There is few report yet concerning ALDH2 genotype and HCC, and some preliminary reports ${ }^{10,11)}$ showed no association. The small sample size in the present study prohibited a multivariate analysis and further study is still necessary to elucidate etiologic interactions among potential factors for developing HCC. 


\section{ACKNOWLEDGEMENTS}

This work was partly supported by Grant-in-Aid for Cancer Research from the Ministry of Health and Welfare, by Grant-in -Aid for Scientific Research (C) from the Ministry of Education, by a grant from Fukuoka Cancer Society and by a grant from Kurume City. The authors are greatly indebted to Dr. Koichi Ide, St. Maria Hospital, Dr. Hirofumi Fukushima, Kurume Daiichi Hospital, and Ms. Toshiko Tsuruta and Ms. Noriko Hashimoto for their assistance.

\section{REFERENCES}

1. Morabia A, Stellman SD, Wynder EL. Smoking prevalence in neighborhood and hospital controls : Implication for hospital-based case-control studies. J Clin Epidemiol, 1996; 49: 885-889.

2. Fukuda K, Shibata A, Hirohata I, et al. A hospital-based case-control study on hepatocellular carcinoma in Fukuoka and Saga prefectures, northern Kyushu, Japan. Jpn J Cancer Res, 1993; 84: 708-714.

3. Nishiyori A, Fukuda K, Itoh K, Kato H. Genotype-phenotype agreement of aldehyde dehydrogenase 2 in 120 healthy Japanese. Kurume Med J, 1994; 41: 117-121.

4. The Liver Cancer Study Group of Japan. The 12th Report of Follow-up Study on Primary Liver Cancer in Japan. The Liver Cancer Study Group of Japan, Kyoto, 1996 (in Japanese).
5. Setoguchi Y, Sakai T. Epidemiological study of antiHCV antibodies in Saga district, Jpn J Clin Med,1991; 49: 383-388 (in Japanese).

6. Wacholder S, Silverman DT, McLaughlin JK, Mandel JS. Selection of controls in case-control studies. Am J Epidemiol, 1992; 135: 1029-1041.

7. Westberg VS. Alcohol measuring scales may influence conclusions about the role of alcohol in human immunodeficiency virus (HIV) risk and progression to acquired immunodeficiency syndrome (AIDS). Am J Epidemiol, 1992; 135: 719-725.

8. Liu S, Serdula MK, Byers T, et al. Reliability of alcohol intake as recalled from 10 years in the past. Am J Epidemiol 1996; 143: 177-186.

9. Hirohata I, Fukuda K, Shibata A. et al. Reproducibility of reports of past history, smoking, drinking and dietary habits obtained by a personal interview for a case-control study on liver cirrhosis and hepatocellular carcinoma. Kurme Med J, 1991; 38: 243-249.

10. Tsutsumi M, Takase S, Takada A. Genetic factors related to the development of carcinoma in digestive organs in alcoholics. Alcohol \& Alcoholism, 1993; 28: 21-29.

11. Takeshita T, Imayoshi S, Satoh S, Morimoto K. Effects of the low $\mathrm{Km}$ aldehyde dehydrogenase polymorphism and alcohol drinking habits on the development of liver cancer in Japanese. (read at) The XIV International Scientific Meeting of the International Epidemiological Association, Nagoya, August 1996. 